\title{
Spectral-temporal dynamics of multipulse mode-locking
}

\author{
Ying Yu, Bowen Li, Xiaoming Wei, ${ }^{\text {a) }}$ Yiqing Xu, Kevin K. M. Tsia, and Kenneth K. Y. Wong ${ }^{\text {b) }}$ \\ Department of Electrical and Electronic Engineering, The University of Hong Kong, Pokfulam Road, \\ Hong Kong, China
}

(Received 6 March 2017; accepted 4 May 2017; published online 17 May 2017)

\begin{abstract}
In addition to stable pulse generation, passively mode-locked fiber lasers can easily run into an unstable regime of multipulse mode-locking. The birth and dynamic behaviors of multipulse modelocking so far have rarely been experimentally explored, particularly in the spectral domain. In this letter, several kinds of multipulse spectral-temporal dynamics of a passively mode-locked fiber laser are observed in a single-shot manner, e.g., energy quantization, self-phase modulation spectral broadening, wavelength shifting, spectral interfering, and ultraweak pulse interaction. This study is enabled by the high temporal resolution of spectral-temporal technology, i.e., $50 \mathrm{ps}$ in the time domain and tens of nanoseconds in the spectral domain. Moreover, a wide observing time span of our spectral-temporal analyzing system, i.e., $>6 \mathrm{~ms}$ - equivalent to 100000 round trips, enables evolutionary characterization of individual pulses. The results will have a significant impact on optimizing the performance of mode-locked fiber lasers and understanding the nonlinear physics in a dissipative system. Published by AIP Publishing. [http://dx.doi.org/10.1063/1.4983718]
\end{abstract}

Fiber lasers have played an increasingly important role in various areas, including biological imaging, ${ }^{1-3}$ optical communication, ${ }^{4}$ and optical spectroscopy, ${ }^{5}$ due to their advantages such as small footprints, high energy, and extensive wavelength ranges. ${ }^{6-8}$ With many longitudinal modes confined in a small core (few microns), fiber lasers can also generate extraordinary laser dynamics that differs from their normal operations, for example, pulse burst, ${ }^{9}$ Q-switching, ${ }^{10}$ optical rogue waves, ${ }^{11,12}$ and so on. In passively mode-locked lasers, in addition, a more complex system can arise from the interplay of gain, loss, dispersion, and nonlinearity. ${ }^{13}$ Consequently, passive mode-locking, no matter what underlying mechanism behind is, can easily run into the multipulse state, which has been widely observed in various platforms, e.g., saturable absorber mode-locked Ti: sapphire lasers, ${ }^{14}$ Kerr-lens mode-locked Ti: sapphire lasers, ${ }^{15}$ and nonlinear polarization rotation (NPR) mode-locked fiber lasers. ${ }^{16}$ In the anomalous-dispersion regime, particularly, hundreds of modelocked pulses can be generated and coexisted in the same round trip. ${ }^{17}$ Although the crowded pulse cluster can produce fruitful optical interactions, e.g., soliton molecules and soliton rain, ${ }^{18,19}$ it is historically a detrimental phenomenon to the stability of mode-locking, i.e., undermining the fundamental mode-locking. It even introduces intensity fluctuations during the sample illumination and detection in applications of bioimaging, which can be a safety concern.

A further understanding of multipulse mode-locking is required to push its optimal operation. Several theoretical attempts have been made to interpret the mechanism behind multipulse mode-locking, e.g., peak power clamping, ${ }^{20}$ gain bandwidth limited pulse splitting, ${ }^{21}$ and nonlinear cavity feedback. ${ }^{22}$ In terms of experimental investigations, however, it is seriously limited by the poor temporal resolution of conventional spectroscopic techniques, which thus hinders

\footnotetext{
${ }^{a)}$ Email: xmwei@eee.hku.hk

${ }^{b}$ kywong@eee.hku.hk
}

the spectral-temporal study of individual pulses. Therefore, tracking the behavior of a single pulse within the multipulse cluster has been mostly conducted by numerical simulations and temporal observation. ${ }^{20,23,24}$

In order to experimentally observe multipulse dynamics, an ultrafast analyzing technique is necessary because the spectral as well as temporal information of the multipulse cluster can rapidly change, e.g., in the order of nanoseconds-the round-trip time $\left(T_{r t}\right)$. Dispersive Fourier transform (DFT) is a single-shot spectrum analyzing technology that enables a frame rate of up to tens of $\mathrm{MHz} .{ }^{25-27}$ As the core of the DFT technique, the group-velocity dispersion (GVD) from dispersive media is used to perform wavelength-to-time mapping for a single pulse. It is thus able to capture a singleshot spectrum at a temporal resolution of a few nanoseconds as well as a spectral resolution of tens of picometers. ${ }^{28}$ As it has been well adopted to study laser dynamics and supercontinuum generation, ${ }^{28-32}$ it should also be an effective tool for understanding the multipulse mode-locking behavior, which, however, is yet to be exploited.

In this letter, we utilize DFT as a part of a single-shot spectral-temporal analyzer to investigate the single-pulse behaviors of the multipulse cluster in a standard passively mode-locked fiber laser. The temporal and spectral characteristics of individual pulses are carefully studied at high temporal and spectral resolutions. Consequently, interesting dynamic phenomena of multipulse mode-locking are observed. The results can broaden the horizon of multipulse mode-locking in addition to previous theoretical and numerical investigations.

Figure 1 shows the overview of the experimental setup for the spectral-temporal study of multipulse mode-locking in a single-shot manner. A passively mode-locked fiber laser using the NPR mode-locking mechanism was employed, as illustrated on the left hand side. A 980-nm laser diode (II-VI LC96AA74P-20) served as a pump source, which was coupled into the cavity via a four-port optical integrated module 


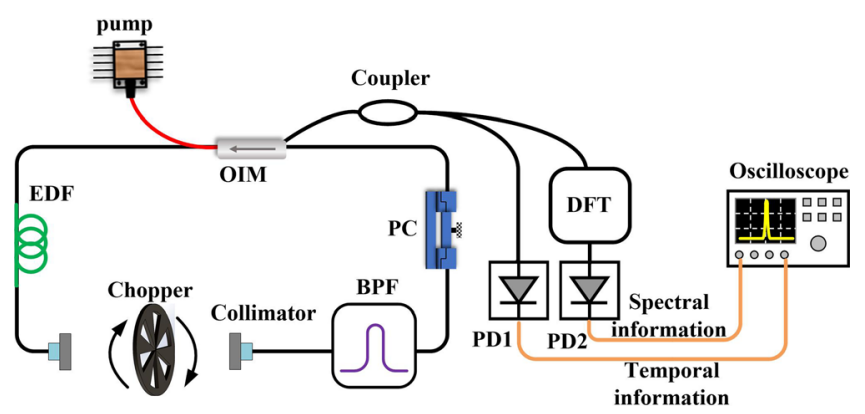

FIG. 1. Experimental setup of the spectral-temporal study of multipulse mode-locking. OIM, optical integrated module; EDF, erbium-doped fiber; $\mathrm{BPF}$, band pass filter; PC, polarization controller; PD, photodetector.

(OIM). The OIM integrated three components into a single unit: (1) wavelength-division multiplexer (WDM) used to couple the 980-nm pump into the cavity; (2) polarizationsensitive isolator used for unidirectional operation and polarization selection, which was a key device to ensure NPR mode-locking by cooperating with a polarization controller (PC); (3) coupler, which was used to extract $10 \%$ of the resonating power out of the cavity for spectral-temporal analysis. The gain medium was a $0.6-\mathrm{m}$ erbium-doped fiber (EDF, Thorlabs ER80-8/125). Other parts of the cavity were made of single-mode fibers (SMFs) and a short section of the freespace path. The total cavity length was about $7 \mathrm{~m}$, corresponding to a repetition rate of 29.2 MHz. A 4-nm band pass filter (BPF) centered at $1540 \mathrm{~nm}$ was used to control the lasing wavelength and facilitate mode-locking. To control the onset of mode-locking, a fast optical chopper system (Thorlabs MC2000-EC) was placed in the free-space section of the fiber cavity. After extracting from the cavity, the laser signal was split into two branches via a 50/50 fiber coupler for spectral and temporal analyses, respectively. To obtain temporal information, the optical signal was directly received by a high-speed photodetector (PD, 32-GHz, HP 83440D) and digitized by a multichannel real-time oscilloscope (Lecroy SDA $820 \mathrm{Zi}-\mathrm{B}, 20 \mathrm{GHz}$ ) at a sampling rate of $40 \mathrm{GS} /$ $\mathrm{s}$, yielding a temporal resolution of about $50 \mathrm{ps}$. The realtime oscilloscope provided a memory depth of up to 256 Mpts for each channel, i.e., corresponding to a continuous viewing span of $6.4 \mathrm{~ms}$ at a sampling rate of $40 \mathrm{GS} / \mathrm{s}$. For interpreting single-shot spectral information, the second branch mapped the optical spectrum of each optical pulse into the time domain through the DFT unit, which actually was a spool of dispersion-compensating fibers (DCF, $10.7 \mathrm{~km}$ in length). The DCF provided a GVD of up to $-0.87 \mathrm{~ns} / \mathrm{nm}$, yielding a spectral resolution of $0.14 \mathrm{~nm} .{ }^{33}$ After converting into electrical signals via another PD (15 GHz, HP 11982A), the single-shot spectral information was finally digitized by another channel of the same real-time oscilloscope. It is noted that the signal digitization of both the channels was synchronized by a trigger signal, while the time delay between them (about $52.07 \mu \mathrm{s}$ ) was compensated by data post-processing.

Basically, the single-pulse mode-locking operation of the fiber laser was self-started by simply increasing the pump power beyond $80 \mathrm{~mW}$ at a proper PC setting. To observe the birth of multipulse mode-locking, the pump power was appropriately increased. Rather than rebooting the pump, which typically takes seconds, the mode-locking was initiated by blocking (OFF) and subsequently unblocking $(\mathrm{ON})$ the cavity through the fast chopping wheel $(254 \mu \mathrm{m}$ in thickness). To maximize the opening speed of the laser beam, it can be focused onto the rotating wheel. In this way, the opening time of the laser beam can be reduced down to tens of nanoseconds, comparable to the round-trip time of the fiber cavity. In every transition from the OFF to ON state, the fiber laser can onset a mode-locking process. In the meantime, the chopper also generated a trigger which can synchronize the data acquisition. A 6.4-ms data stream (256 Mpts) in both the time and spectral domains was captured for each birth of multipulse mode-locking. It can thus cover the entire birth evolution of mode-locking, i.e., from noise to continuous mode-locking, which typically takes hundreds of $\mu$ s. To analyze the spatio-temporal behavior of multipulse mode-locking, the acquired data from the temporal channel were segmented according to the round-trip time of the fiber cavity, i.e., $34.2 \mathrm{~ns}$ in this case, and lined up according to the round-trip number $\left(N_{r t}\right)$. Similar data processing was performed for the spectral channel, except that it was lined up according to the extracting time $\left(N_{r t} \times T_{r t}\right)$, given a wavelength coordinate according to the DFT dispersion amount.

Figure 2 shows the spatio-temporal map (i.e., timedomain evolution) of a multipulse mode-locking. It is clear that six pulses were born at the very beginning, while the leftmost one (denoted as "i" in Fig. 2) vanished after $575 \mu \mathrm{s}$. This actually confirms the energy quantization theory ${ }^{20}$ that a given pump power can only support a specific number (i.e., 5 in this case) of coexisting mode-locked pulses in a dynamic manner, and it is further confirmed by other births of modelocking in a series of experiments. It is also noted that a strong intensity fluctuation, i.e., Q-switching, was present at the early birth stage of each arising pulse, as shown in the bottom right inset of Fig. 2, i.e., the close-up of the birth of the second pulse. After this short period of the Q-switched fluctuation, it turned into mode-locking, as shown in the left inset of Fig. 2. Interestingly, the propagation speed $\left[v_{g}=c / n_{g}(\lambda)\right]$ of optical pulses at the beginning was different from that of stable mode-locking, i.e., manifested by a bending trajectory, as shown in the bottom right inset of Fig. 2 . This arises from the center wavelength shifting during the transition into mode-locking, which will be further verified by the single-pulse spatio-spectral evolution and demonstrated later. Among those survived pulses, the slow intensity

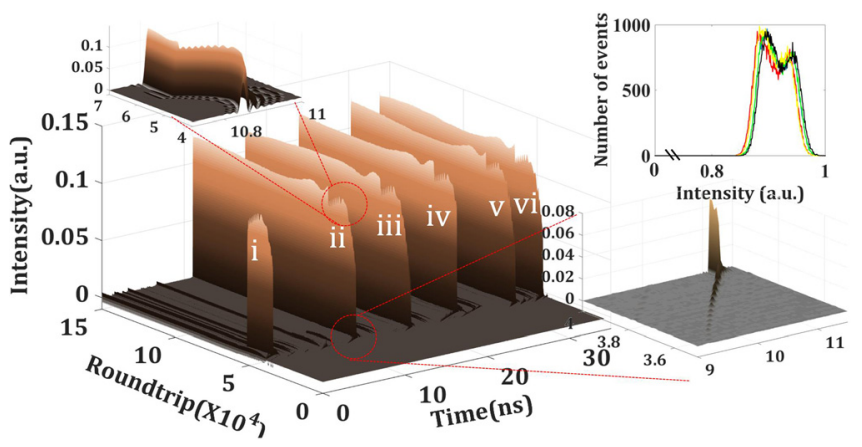

FIG. 2. The spatio-temporal evolution of multipulse mode-locking. The left inset shows the close-up of the beginning of mode-locking. The bottom right inset shows the close-up of the Q-switched fluctuation. The top right inset shows the intensity histograms of individual survived pulses. 
variation existed over the entire spatio-temporal evolution. In order to qualitatively explore the intensity evolution, the statistical intensity histograms of each evolving pulse over 80000 round-trips were calculated and are shown in the top right inset of Fig. 2. They exhibit similar histogram shapes for each pulse, i.e., a double-peak distribution. This implies that each pulse has two main intensity levels over the entire evolution, which can be attributed to the gain competition between them.

Thanks to the high temporal resolution of single-shot DFT spectroscopy, the spatio-spectral evolution of individual pulses among the pulse cluster can be investigated. Figure 3(a) illustrates the spatio-spectral map of the second pulse (ii). It is clear that the optical spectrum of mode-locked pulse (ii) was evolved from a narrow one centered at around $1540.8 \mathrm{~nm}$. Then, it was followed by a spectral broadening process as shown in the inset of Fig. 3(a). There were also strong fluctuations right before broadband mode-locking, as indicated by the yellow and white dashed circles in the inset of Fig. 3(a). More interestingly, spectral oscillation was also present in the last two strong shocks, as indicated by white and red dashed circles. This is a signature of the self-phase modulation (SPM) effect, which plays a dominant role in NPR mode-locking ${ }^{34}$ as well as supercontinuum generation ${ }^{35}$ for initial spectrum broadening. Particularly, the " $\mathrm{M}$ " shape spectral broadening is a result of the 1.5- $\pi$ maximum nonlinear phase shift induced by SPM. ${ }^{36}$ After this initial SPM spectral broadening, it was rapidly evolved into a smooth broad spectrum, which might be attributed to other nonlinear effects, e.g., cross-phase modulation (XPM), stimulated Raman scattering (SRS), and four-wave mixing (FWM). It is also noticed that the center wavelengths are different in the regimes before and after mode-locking, as shown in the close-up of Fig. 3(a), which can be attributed to the asymmetrical spectral broadening and agrees with the finding of Ref. 28. It is also the origin of the bending trajectory as shown in Fig. 2.

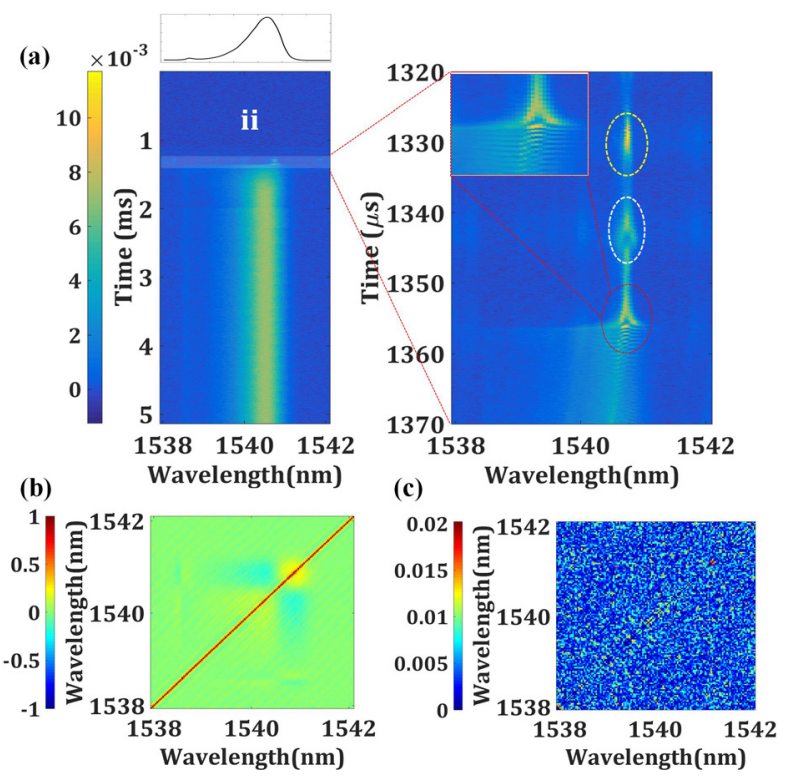

FIG. 3. (a) The spatio-spectral evolution of pulse (ii). The top inset shows the average spectrum. (b) The SIC map of pulse (ii). (c) The SIC difference between pulses (ii) and (iii).
In the stable mode-locking regime, i.e., after $2.4 \mathrm{~ms}$ as shown in Fig. 3(a), the 2D spectral intensity correlation $(\mathrm{SIC})^{37}$ map of these survived pulses can be calculated, which benefits from the high temporal and spectral resolution of DFT technology. The SIC function ranges from -1 to 1: a synchronous intensity variation of two wavelengths corresponds to a positive SIC, which otherwise yields a negative one. Figure 3(b) shows the 2D SIC map of pulse (ii), while the other four survived pulses show a consistent pattern (not shown here). The SIC difference between pulse (ii) and others was calculated, e.g., Fig. 3(c) shows the SIC difference between pulses (ii) and (iii). The maximum absolute differences over the whole 2D SIC map were measured to be $0.0202,0.0248,0.0238$, and 0.0254 for pulses (iii-vi), respectively. The results suggest that both the temporal and spectral evolutions of individual pulses in the multipulse modelocking exhibit similar characteristics.

Among different births of multipulse mode-locking, it is noticed that the pulse distribution, mainly the spacing between pulses, varied from case to case, even under the same experimental setting. However, for a given pump power, the total number of survived pulses remains the same for every birth of the pulse cluster, which agrees with the numerical finding. ${ }^{20}$ More interestingly, pulse interference in the spectral domain was observed in certain building-up journey of multipulse mode-locking, as shown in Fig. 4. In the spatio-temporal evolution shown in Fig. 4(a), four pulses are well separated. In the spectral domain, pulse (iii) actually delivers spectral dynamics that differs from others, as shown in Fig. 4(b). Strong shocks associated with SPM spectral broadening also appear before stable mode-locking. However, unlike the case shown in Fig. 3(a), spectral fringes are presented right after spectral broadening, which implies that pulse (iii) is actually two interfering pulses. The distinguished spectral fringe indicates a good coherence between the interfering pulses, as shown in the top inset of Fig. 4(b). After mode-locking, the stability of interfering pulses might be a concern since the SIC map of the interfering pulses, as shown in Fig. 4(c), exhibits a very different pattern compared to the case of Fig. 3(b).

To give more details, the temporal separation evolution between the interfering pulses was retrieved from the spectral fringes and is shown in Fig. 4(d). It is clear that the interfering pulses weakly repel each other, resulting in a weak drifting of less than $10 \mathrm{ps}$ over $2 \mathrm{~ms}$, which is equivalent to a change of about $2 \mathrm{~mm}$ over a traveling distance of $400 \mathrm{~km}$. This indicates that there is only an ultraweak interaction between the interfering pulses, i.e., weak repulsive optical force in this case. The mechanism behind can be mainly attributed to the electrostriction, an effect that the electric field of a short pulse (e.g., the leading pulse in this interfering case) can deform the optical fiber and emit an acoustic wave, which is back-and-forth reflected between the fiber cladding boundaries in a period of about $20 \mathrm{~ns}$ for a $125-\mu \mathrm{m}$ fiber ${ }^{38,39}$ and results in a small time-varying change of the refractive index. Thus, the following pulse can experience a small center wavelength shifting. As the velocity of the optical pulse is wavelength-dependent due to the nature of dispersion, the following pulse can either accelerate or decelerate (i.e., this case). Indeed, some other origins of pulse interactions have also been discussed for mode-locked fiber cavities, such as 

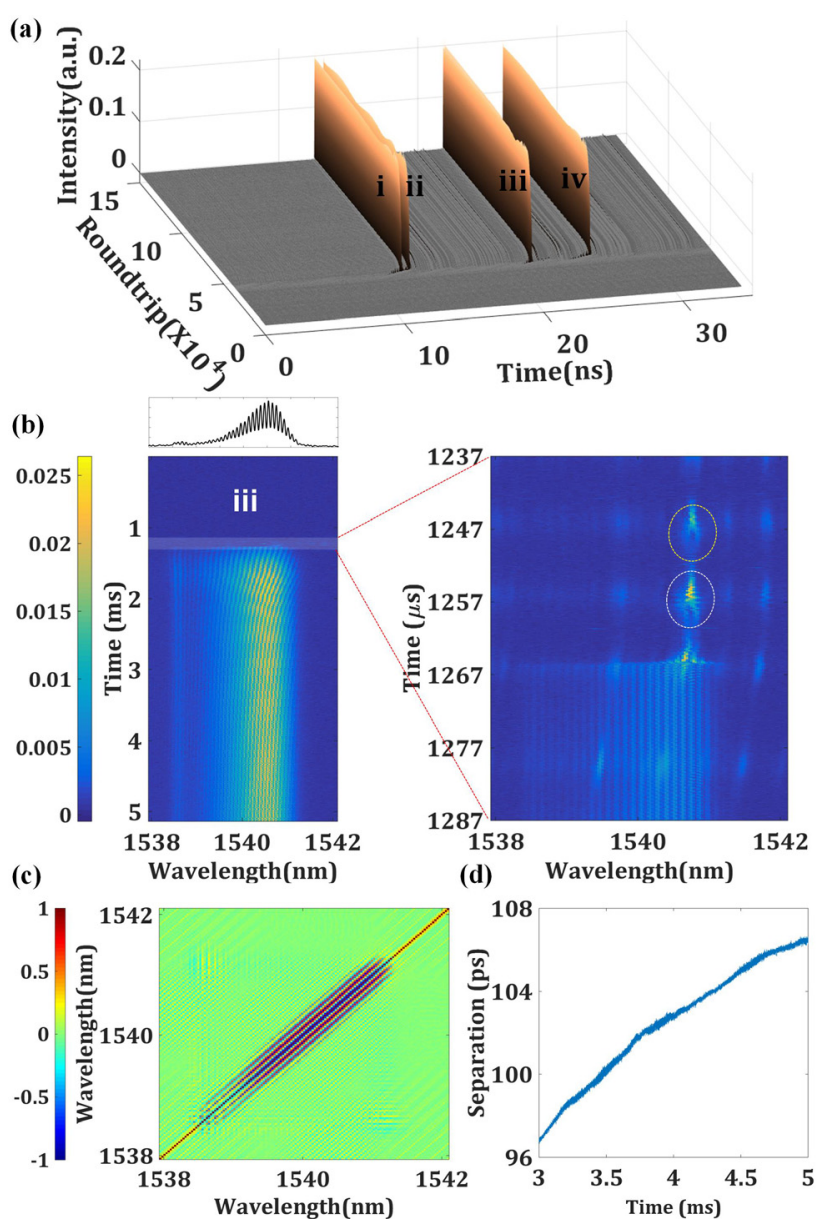

(d) Wavelength $(\mathrm{nm})$

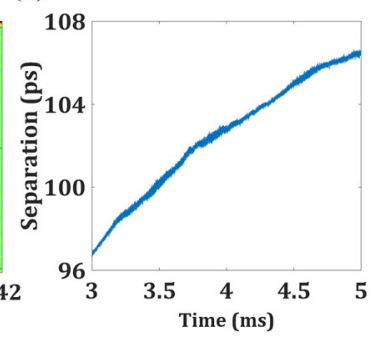

FIG. 4. (a) The spatio-temporal evolution of interfering multipulse modelocking. (b) The spatio-spectral evolution of interfering pulses (iii). The top inset shows one frame for example. (c) The SIC map of the interfering pulses. (d) The temporal separation evolution between interfering pulses.

global soliton interactions through an additional unstable continuous-wave (CW) background, ${ }^{40,41}$ local soliton interactions via dispersive wave emission, ${ }^{42}$ and direct soliton interactions. ${ }^{43}$ However, they are not involved in the current case, and the reasons include: (1) there is no additional $\mathrm{CW}$ component shown in the mode-locked spectrum, which has also been verified by standard spectroscopic tools; (2) the operating bandwidth of the current laser cavity is confined by a 4$\mathrm{nm}$ filter, and the zero-dispersion wavelengths (ZDWs) of single-mode fibers constructed the cavity are far away from the operating wavelength, i.e., $1.3 \mu \mathrm{m}$ and $1.5 \mu \mathrm{m}$. Thus, phase matching for dispersive wave generation is very difficult; $^{44}$ (3) the direct soliton interaction can only occur for close pulses, typical separated by a few picoseconds (i.e., several times wider than their pulse width).

It should be pointed out that stable pulsing is not guaranteed in each onset of mode-locking. In some cases, no stable pulse can be observed, as exemplified in Fig. 5, which indeed exhibits the complex nature of this nonlinear dissipative system. Similar to the previous cases, right after turning into the ON stage, the arising pulses exhibit strong fluctuations in the time domain. Instead of generating stable broadband pulses as that of previous cases, i.e., Figs. 2 and 4(a), the narrowband pulses last for a relatively long time, and strong interactions occur among them. As can be observed in Fig. 5, there

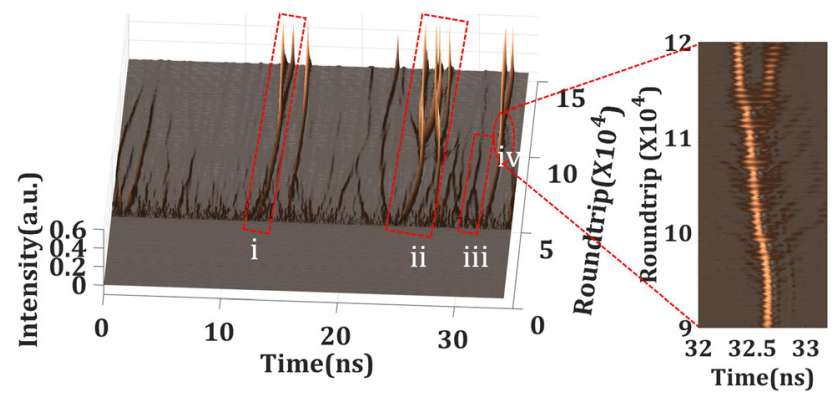

FIG. 5. Interaction between multiple narrowband pulses.

are several kinds of interactions, which are indicated by the dashed square boxes and the close-up. For example, pulse splitting occurred in (i) and (iv). Particularly in (iv), the original pulse splits into several low-intensity unstable pulses over the evolution from $9 \times 10^{4}$ to $12 \times 10^{4}$ roundtrips, while most of them vanish soon after generation. In (ii), two pulses first attract each other until the spacing between them becomes tens of picoseconds, and then, they start to repel each other. In (iii), the temporal separation of two pulses keeps reducing along the propagation, which is similar to the first phase of case (ii), and eventually they merge into a single pulse.

In summary, multipulse dynamics in a standard passively mode-locked fiber laser has been investigated in both the time and spectral domains at high temporal resolutions. The entire birth process of a single pulse in the multipulse mode-locking, including Q-switched fluctuations, transient SPM spectral broadening, wavelength shifting, and ultraweak pulse interactions through electrostriction, has been observed. The pulse intensity consistency and spectral intensity correlation of a single pulse, as well as the spectral interfering, have particularly been studied. These are enabled by the high resolution and long time span (i.e., memory depth) of our spectral-temporal single-shot analyzer. The fruitful findings in this work are believed to broaden the horizon of passive multipulse mode-locking. A better experimental understanding of spectral-temporal fluctuations in passive mode-locking can not only be complementary to the theoretical and numerical predictions but also be very important in optimizing the operations of passive mode-locking for safety-sensitive applications, particularly in the biological areas.

The work described in this paper was partially supported by grants from the Research Grants Council of the Hong Kong Special Administrative Region, China (Project Nos. HKU 17205215, HKU 17208414, and CityU T42-103/16N), National Natural Science Foundation of China (N_HKU712/16), Innovation and Technology Fund (GHP/ 050/14GD), and University Development Fund of HKU.

${ }^{1}$ C. Xu and F. W. Wise, Nat. Photonics 7, 875-882 (2013).

${ }^{2}$ B. E. Bouma, L. E. Nelson, G. J. Tearney, D. J. Jones, M. E. Brezinski, and J. G. Fujimoto, J. Biomed. Opt. 3, 76-79 (1998).

${ }^{3}$ C. W. Freudiger, W. Yang, G. R. Holtom, N. Peyghambarian, X. S. Xie, and K. Q. Kieu, Nat. Photonics 8, 153-159 (2014).

${ }^{4}$ V. Mizrahi, D. J. DiGiovanni, R. M. Atkins, S. G. Grubb, Y. K. Park, and

J. M. P. Delavaux, J. Lightwave Technol. 11, 2021-2025 (1993).

${ }^{5}$ S. T. Sanders, Appl. Phys. B 75, 799-802 (2002). 
${ }^{6}$ K. Tamura, E. P. Ippen, H. A. Haus, and L. E. Nelson, Opt. Lett. 18, 1080-1082 (1993).

${ }^{7}$ C. Jauregui, J. Limpert, and A. Tünnermann, Nat. Photonics 7, 861-867 (2013).

${ }^{8}$ S. D. Jackson, Nat. Photonics 6, 423-431 (2012).

${ }^{9}$ D. V. Churkin, S. Sugavanam, N. Tarasov, S. Khorev, S. V. Smirnov, S. M. Kobtsev, and S. K. Turitsyn, Nat. Commun. 6, 7004 (2015).

${ }^{10}$ M. Delgado-Pinar, D. Zalvidea, A. Díez, P. Pérez-Millán, and M. V. Andrés, Opt. Express 14, 1106-1112 (2006).

${ }^{11}$ D. R. Solli, C. Ropers, P. Koonath, and B. Jalali, Nature 450, 1054-1057 (2007).

${ }^{12}$ C. Lecaplain, P. Grelu, J. M. Soto-Crespo, and N. Akhmediev, Phys. Rev. Lett. 108, 233901 (2012).

${ }^{13}$ P. Grelu and N. Akhmediev, Nat. Photonics 6, 84-92 (2012).

${ }^{14}$ M. J. Lederer, B. Luther-Davies, H. H. Tan, C. Jagadish, N. N Akhmediev, and J. M. Soto-Crespo, J. Opt. Soc. Am. B: Opt. Phys. 16, 895-904 (1999).

${ }^{15}$ J. H. Lin, W. F. Hsieh, and H. H. Wu, Opt. Commun. 212, 149-158 (2002).

${ }^{16}$ A. Komarov, H. Leblond, and F. Sanchez, Opt. Commun. 267, 162-169 (2006).

${ }^{17}$ F. Amrani, A. Haboucha, M. Salhi, H. Leblond, A. Komarov, P. Grelu, and F. Sanchez, Opt. Lett. 34, 2120-2122 (2009).

${ }^{18}$ S. Chouli and P. Grelu, Opt. Express 17, 11776-11781 (2009).

${ }^{19}$ C. Bao, X. Xiao, and C. Yang, Opt. Lett. 38, 1875-1877 (2013).

${ }^{20}$ D. Y. Tang, L. M. Zhao, B. Zhao, and A. Q. Liu, Phys. Rev. A 72, 043816 (2005).

${ }^{21}$ F. X. Kärtner, J. Aus der Au, and U. Keller, IEEE J. Sel. Top. Quantum Electron. 4, 159-168 (1998).

${ }^{22}$ A. Komarov, H. Leblond, and F. Sanchez, Phys. Rev. A 71, 053809 (2005).

${ }^{23}$ S. Chouli and P. Grelu, Phys. Rev. A 81, 063829 (2010).

${ }^{24} \mathrm{P}$. Grelu, Nonlinear Optical Cavity Dynamics: From Microresonators to Fiber Lasers (Wiley, 2015).

${ }^{25}$ D. R. Solli, J. Chou, and B. Jalali, Nat. Photonics 2, 48-51 (2008).

${ }^{26}$ K. Goda, K. K. Tsia, and B. Jalali, Nature 458, 1145-1149 (2009).
${ }^{27}$ K. Goda and B. Jalali, Nat. Photonics 7, 102-112 (2013).

${ }^{28}$ G. Herink, B. Jalali, C. Ropers, and D. R. Solli, Nat. Photonics 10, 321-326 (2016)

${ }^{29}$ B. Wetzel, A. Stefani, L. Larger, P. A. Lacourt, J. M. Merolla, T. Sylvestre, A. Kudlinski, A. Mussot, G. Genty, F. Dias, and J. M. Dudley, Sci. Rep. 2, 882 (2012).

${ }^{30}$ A. F. J. Runge, C. Aguergaray, N. G. R. Broderick, and M. Erkintalo, Opt. Lett. 39, 319-322 (2014).

${ }^{31}$ M. Liu, A. P. Luo, Y. R. Yan, S. Hu, Y. C. Liu, H. Cui, Z. C. Luo, and W. C. Xu, Opt. Lett. 41, 1181-1184 (2016).

${ }^{32}$ M. J. Schmidberger, D. Novoa, F. Biancalana, P. S. J. Russell, and N. Y, Joly, Opt. Express 22, 3045-3053 (2014).

${ }^{33}$ K. K. Tsia, K. Goda, D. Capewell, and B. Jalali, Opt. Express 18, 10016-10028 (2010).

${ }^{34}$ M. Hofer, M. E. Fermann, F. Haberl, M. H. Ober, and A. J. Schmidt, Opt. Lett. 16, 502-504 (1991).

${ }^{35}$ J. M. Dudley, G. Genty, and S. Coen, Rev. Mod. Phys. 78, 1135-1184 (2006).

${ }^{36}$ R. H. Stolen and C. Lin, Phys. Rev. A 17, 1448-1453 (1978).

${ }^{37}$ T. Godin, B. Wetzel, T. Sylvestre, L. Larger, A. Kudlinski, A. Mussot, A. Ben Salem, M. Zghal, G. Genty, F. Dias, and J. M. Dudley, Opt. Express 21, 18452-18460 (2013).

${ }^{38}$ J. K. Jang, M. Erkintalo, S. G. Murdoch, and S. Coen, Nat. Photonics 7, 657-663 (2013)

${ }^{39}$ A. N. Pilipetskii, E. A. Golovchenko, and C. R. Menyuk, Opt. Lett. 20, 907-909 (1995).

${ }^{40}$ D. Y. Tang, B. Zhao, L. M. Zhao, and H. Y. Tam, Phys. Rev. E 72, 016616 (2005).

${ }^{41}$ A. Komarov, K. Komarov, A. Niang, and F. Sanchez, Phys. Rev. A 89, 013833 (2014).

${ }^{42}$ M. Olivier, V. Roy, and M. Piché, Opt. Lett. 31, 580-582 (2006).

${ }^{43}$ D. Y. Tang, W. S. Man, H. Y. Tam, and P. D. Drummond, Phys. Rev. A 64, 33814 (2001).

${ }^{44}$ K. E. Webb, Y. Q. Xu, M. Erkintalo, and S. G. Murdoch, Opt. Lett. 38, $151-153$ (2013) 